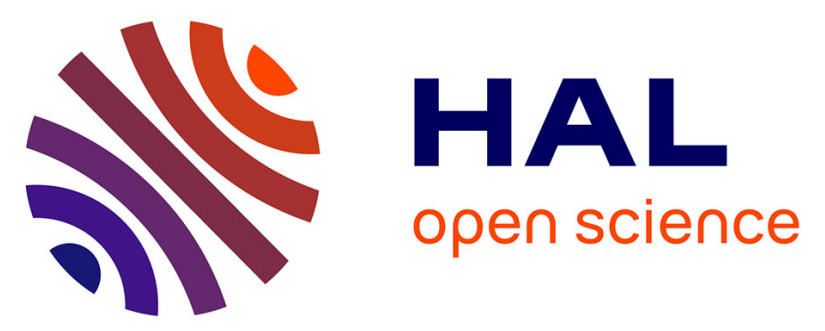

\title{
Reviving the Idea of Incremental Cryptography for the Zettabyte Era Use Case: Incremental Hash Functions Based on SHA-3
}

\author{
Hristina Mihajloska, Danilo Gligoroski, Simona Samardjiska
}

\section{To cite this version:}

Hristina Mihajloska, Danilo Gligoroski, Simona Samardjiska. Reviving the Idea of Incremental Cryptography for the Zettabyte Era Use Case: Incremental Hash Functions Based on SHA-3. International Workshop on Open Problems in Network Security (iNetSec), Oct 2015, Zurich, Switzerland. pp.97111, 10.1007/978-3-319-39028-4_8. hal-01445801

\section{HAL Id: hal-01445801 \\ https://hal.inria.fr/hal-01445801}

Submitted on 25 Jan 2017

HAL is a multi-disciplinary open access archive for the deposit and dissemination of scientific research documents, whether they are published or not. The documents may come from teaching and research institutions in France or abroad, or from public or private research centers.
L'archive ouverte pluridisciplinaire HAL, est destinée au dépôt et à la diffusion de documents scientifiques de niveau recherche, publiés ou non, émanant des établissements d'enseignement et de recherche français ou étrangers, des laboratoires publics ou privés. 


\title{
Reviving the Idea of Incremental Cryptography for the Zettabyte era Use case: Incremental Hash Functions Based on SHA-3
}

\author{
Hristina Mihajloska ${ }^{1}$, Danilo Gligoroski ${ }^{2}$, and Simona Samardjiska ${ }^{1}$ \\ Faculty of Computer Science and Engineering, UKIM, Skopje, Macedonia \\ hristina.mihajloska@finki.ukim.mk, simona.samardjiska@finki.ukim.mk \\ Department of Telematics, NTNU, Trondheim, Norway \\ danilog@item.ntnu.no
}

\begin{abstract}
According to several recent studies, the global IP communication and digital storage have already surpassed the zettabyte threshold $\left(10^{21}\right.$ bytes $)$. The Internet entered the zettabyte era in which fast and secure computations are important more than ever. One solution for certain types of computations, that may offer a speedup up to several orders of magnitude, is the incremental cryptography. While the idea of incremental crypto primitives is not new, so far its potential has not been fully exploited. In this paper, we define two incremental hash functions $i$ SHAKE128 and $i$ SHAKE256 based on the recent NIST proposal for SHA-3 Extendable-Output Functions SHAKE128 and SHAKE256. We describe two practical implementation scenarios of the newly introduced hash functions and compare them with the already known treebased hash scheme. We show the trends of efficiency gains as the amount of data increases in comparison to the standard tree-based incremental schemes. Our proposals $i$ SHAKE128 and $i$ SHAKE256 provide security against collision attacks of 128 and 256 bits, respectively.
\end{abstract}

Keywords: Incremental hashing, SHA-3, Shake128, Shake256, iShake128, iShake256, Zettabyte era

\section{Introduction}

The idea of incremental hashing was introduced by Bellare, Goldreich and Goldwasser in [4] and improved later in [5]. Incremental hashing can be achieved also by using Merkle trees [15] as it is discussed for example in [7]. In a nutshell, the idea of incremental hashing is that if we have already computed the hash value of some document, and this document is modified in one part, then instead of re-computing the hash value of the whole document from scratch, we just need to update it, performing computations only on the changed part of the document. In this way, 
incremental hashing of closely related documents, compared to classical hashing, offers speed gain up to several orders of magnitude. Yet, so far, the concept has not been particularly well accepted by the community nor the industry, and this is mainly due to the following two reasons: 1 . The security level of the incremental hash functions of Bellare et al. $[4,5]$ is detached from the size of the produced hash value, since a standard security of 128 bits requires outputs of several thousand bits. This is very different from the ordinary cryptographic hash functions such as SHA1, SHA-2, SHA-3, where the size of the hash value corresponds to the claimed bit-security level of the hash function. 2. The implementations of these hash functions require expensive modular operations over large prime integers, which makes them one or more orders of magnitude slower than the ordinary cryptographic hash functions.

In the meantime the size of the digital universe has already surpassed 4.4 zettabytes and the projections are that by 2020, it will reach 44 zettabytes [10]. In another report, the Cisco Visual Networking Index [9] predicted that "the annual global IP traffic will pass the zettabyte threshold by the end of 2015, and will reach 1.4 zettabytes per year by 2017." Additionally, the data storage cost according to the latest reports is no longer an issue (see [3]). Hence, the sheer scale of data mentioned, already calls for new solutions that will use the paradigm of incrementality.

Let us consider the use case scenario of sensor networks where data comes from the nodes whose data rates rapidly increase as sensor technology improves and as the number of sensors expands [12]. A typical representative for this scenario is environmental sensor networks used for natural disaster prevention or weather forecasting. In these cases, all data that is collected from different sensors should be publicly available, with data integrity guaranteed by digital signatures from a trusted party. Thus, data hashing is unavoidable, and as the dataset is being updated, the hash value should be recomputed. Normally, the update of such datasets is done by appending new data or by changing a small part of the existing dataset. As the size of the dataset grows, (and can reach hundreds of terabytes [18]), recalculating the hash value of the entire dataset can become notoriously demanding in terms of both time and energy. An incremental update, on the other hand, can reduce the recalculation of the hash value to the minimum, and only of the parts of the dataset that have changed, or have been appended.

Another use case scenario where updates come in the form of insertions of new elements or modifications of existing data are distributed storage systems for managing structured data, such as Cloud Bigtable by Google [8]. It is designed to scale to a very large size, like petabytes of 
data across thousands of commodity servers. Its data model uses Google SSTable file format to internally store data. Each SSTable contains a sequence of blocks typically of $64 \mathrm{~KB}$ in size and every block has its own unique index that is used to locate the block. Using this kind of file formats where blocks have its unique numbers, incremental hashing can be successfully implemented despite the variable-size setting: In addition to the update operation, in order to perform incremental hash calculations, additional insert and delete operations should be introduced.

The trade-off between re-hashing and incremental hashing is simply in the storing of additional data overhead in order to get computation speed. Instead of rehashing the whole file (for example 1GB), with the incremental hashing you just need to re-hash a small part of it (for example $1 \mathrm{MB}$ ), but the price is to keep a data overhead used in the process of incremental hashing.

The initial idea for an incremental hash function based on the recent NIST proposal for SHA-3, Extendable-Output Functions SHAKE128 and SHAKE256 [17] was presented at the NIST SHA-3 2014 Workshop [11]. We improve that proposal, define two practical implementation instances: iSHAKE-128 and iSHAKE-256 and compare them with already known incremental tree-based hash schemes.

The paper is organized as follows: In Section 2 we give mathematical preliminaries and definitions about incremental hash functions. In Section 3 we give an algorithmic description of incremental operations for two practical settings. After that, in Section 4, we define two incremental hash functions with security levels of 128 and 256 bits. Comparison analysis between our proposals and incremental tree-based hash scheme is given in Section 5. Finally, we conclude our paper in Section 6 with recommendations on where and how to use our incremental hash functions.

\section{Mathematical preliminaries}

\subsection{Incremental hash functions}

We will use the following definition for an incremental hash function adapted from [5, Sec. 3.1]:

Definition 1. Let $h:\{0,1\}^{b} \rightarrow\{0,1\}^{k}$ be a compression function that maps $b$ bits into $k$ bits. Let the message $M$ be represented as a concatenation of $n$ blocks, where $n<N$ for some predefined number $N$ which is larger than the number of blocks in any message we plan to hash, i.e., $M=M_{1}\left\|M_{2}\right\| \ldots \| M_{n}$. The size of each block $M_{i}$ is determined by the following relation: $\left|M_{i}\right|=b$-Length $\left(I D_{i}\right)$, where $I D_{i}$ is a unique identifier for the block $M_{i}$. 
For each block $M_{i}, i=1, \ldots, n$, append $I D_{i}$ to get an augmented block $\overline{M_{i}}=M_{i} \| I D_{i}$. For each $i=1, \ldots, n$, apply $h$ to $\overline{M_{i}}$ to get a hash value $y_{i}=h\left(\overline{M_{i}}\right)$. Let $(G, \odot)$ be a commutative group with operation $\odot$ where $G \subseteq\{0,1\}^{k}$. Combine $y_{1}, \ldots, y_{n}$ via a combining group operation $\odot$ to get the final hash value

$$
y=y_{1} \odot y_{2} \odot \ldots \bigodot y_{n} .
$$

Denote the incremental hash function as:

$$
\begin{aligned}
& \text { the incremental hash function as: } \\
& y(M)=\mathrm{HASH}_{\langle G\rangle}^{h}\left(M_{1}\left\|M_{2}\right\| \ldots \| M_{n}\right)=\bigodot_{i=1}^{n} h\left(M_{i} \| I D_{i}\right)
\end{aligned}
$$

Since the group $(G, \odot)$ is commutative, the computation is parallelizable too. In such a case, the combining group operation $\odot$ is commutative and invertible, and increments are done as follows. If block $M_{i}$ changes to $M_{i}^{\prime}$, then the new hash value is computed as $y\left(M^{\prime}\right)=$ $y(M) \bigodot^{-1} h\left(\overline{M_{i}}\right) \bigodot h\left(\overline{M_{i}^{\prime}}\right)$ where $\bigodot^{-1}$ denotes the inverse operation in the group $(G, \odot)$ and $y(M)$ is the old hash value. The cost of an increment operation is two hash computations and two operations in $G$.

The choice of good combining operation is important for both security and efficiency. In [5] there are four different hash function families depending on the combining operation. In XHASH, the combining operation is bitwise XOR. The multiplicative hash, MuHASH uses multiplication in a group where the discrete logarithm problem is hard. AdHASH stands for hash function obtained by setting the combining operation to addition modulo a sufficiently large integer, and LtHASH uses vector addition. Out of these four, the scheme XHASH is not secure. The authors of [5] estimated that the hash value of size $\approx 1024$ bits would suffice for the security level of $2^{80}$. However, Wagner in [19] showed that using a generalized birthday attack, these parameters are breakable, implying that the size of the hash values should be much bigger (for standard security levels, even up to tens of thousands of bits). Wagner also showed how to solve the $n$-sum problem for certain operations (a special case of the balance problem), with time and space complexity of $O\left(n \cdot 2^{\frac{k}{1+\lg [n]}}\right)$ using lists of size $2^{\frac{k}{1+\lg [n]}}$ elements. More precisely, Wagner [19] showed the following:

Proposition 1. Let $\mathrm{HASH}_{\langle G\rangle}^{h}$ be an incremental hash function defined by Definition 1. For any $Y \in\{0,1\}^{k}$ the complexity of finding a preimage message $M=M_{1}\left\|M_{2}\right\| \ldots \| M_{n}$ of length $n \leqslant N$ blocks such that $Y=$ $\operatorname{HASH}_{\langle G\rangle}^{h}(M)$ is:

$$
\min _{n \leqslant N} O\left(n \cdot 2^{\frac{k}{1+\lg [n]}}\right)
$$

If the length of the messages is not restricted, then the minimum in equation (2) is achieved for messages of $n=2^{\sqrt{k}-1}$ blocks. 
So, 10-15 years ago, the lack of an urgent need to hash extremely big files, as well as the difference between the hash sizes of classical hash functions (160 - 512 bits) versus the hash sizes in the incremental case (2500 - 16000 bits due to Wagner's result [19]), killed the attractiveness of the concept of incremental hashing. However, there are new trends and a new reality. In particular: the latest SHA-3 standard allows arbitrary hash sizes [17]; the need for incremental digesting of big files is increasing and the cost of storing longer hash values is decreasing. These are the main reasons why we revive the idea of incremental hashing in this paper.

\section{Incremental hashing scheme}

We will instantiate the incremental hash function from Definition 1 in two practical settings: fixed-size data and variable-sized data. In the fixed-size data setting, the data that needs to be hashed has a predetermined fixed size, and thus the total number of data blocks is fixed. The real use case scenarios can be found in cloud services (Images of Virtual Machines [1,2], cloud storage [13]), digital movies distributions [16], collecting data from sensor networks and many more. In the fixed-size data scenario, the incremental operations that need to be implemented are: block replacement (replace operation) and block appending. The other setting is a variablesize data, such as managing structured data, where additionally the incremental operations for insertion (insert operation) and deletion (delete operation) of a block should be supported. In order to implement these operations, we will use dynamic data structures.

For both of the aforementioned scenarios, the basic algorithmic description is given in Algorithm 1. The underlying hash primitive and combining operation in the algorithm are the following:

Underlying hash function. The concrete hash function $h$ has to map $b$ bits into $k$ bits ( $k$ is a multiple of 64 ), $h:\{0,1\}^{b} \rightarrow\{0,1\}^{k}$. Typical cryptographic hash functions such as SHA-1 or SHA-2 output a short hash value of 160 or 256 or 512 bits. However, for achieving security levels of 128 or 256 bits we need the value of $k$ to be more than 2000 bits. We use the recently proposed Extendable-Output Functions SHAKE128 and SHAKE256 defined in the NIST Draft FIPS-202 [17]. Definition and security analysis are given in Section 4.

Combining operation. For the compression function $h:\{0,1\}^{b} \rightarrow$ $\{0,1\}^{k}$ where $k$ is a multiple of 64 bits i.e. $k=64 \cdot L$, we use wordwise addition in the commutative group $\left(\left(\mathbb{Z}_{2^{64}}\right)^{k / 64}, \bigoplus_{64}\right)$, since it is a very efficient operation on the modern 64 -bit CPUs. The operation $\bigoplus_{64}$ represents 64 -bit word-wise addition of $k / 64$ words, and $\square_{64}$ the inverse operation of word-wise subtraction of $k / 64$ words. 


\begin{tabular}{|c|}
\hline Algorithm 1 - Incremental hash function \\
\hline $\begin{array}{l}\text { Input. A sequence of blocks } M_{1}, M_{2}, \ldots, M_{n} \text {, where each } M_{i} \text { has a fixed size of } \\
b \text { - Length }(I D) \text { bits. }\end{array}$ \\
\hline Output. $k$ bits of hash output. \\
\hline $\begin{array}{l}\text { 1. For each block } M_{i}, i=1, \ldots, n \text {, append } I D_{i} \text { to get an augmented block } \overline{M_{i}}= \\
M_{i} \| I D_{i} ; \\
\text { 2. For each } i=1, \ldots, n \text {, apply } h \text { to the blocks } \overline{M_{i}} \text { to get a hash value } y_{i}=h\left(\overline{M_{i}}\right) \\
\text { 3. Combine } y_{1}, \ldots, y_{n} \text { via the group operation } \bigoplus_{64} \text { to get the final hash value: } \\
\qquad y=y_{1} \square_{64} y_{2} \square_{64} \ldots \square_{64} y_{n} .\end{array}$ \\
\hline 4. Output $y$ and store it. \\
\hline
\end{tabular}

Fig. 1. An algorithm for incremental hash function. Note that when we deal with the fixed size data $I D_{i} \equiv\langle i\rangle$ and for variable size setting it is $I D_{i} \equiv\left(B N_{i}, p t r_{B N_{i}}\right)$

Using appropriate parameter values for the formulations above, we have two practical settings:

1. Fixed-size data. Hashing data which has a predetermine fixed size. The total number of data blocks is fixed, or can be changed by appending new blocks.

Block indexing. The data $M$ is virtually divided into a fixed number of blocks $M_{1}, \ldots, M_{n}$. In this case, each block $M_{i}$ has index $i$ and its 64-bit binary encoding represents its unique identifier $I D_{i} \equiv\langle i\rangle$. This virtual division of data is shown in Figure 2 .

Incremental update operation. Once the hash function is applied on $M$, there is no need to repeat the same procedure for the whole $M$, but we can apply an incremental update operation. In this case the only update operation is the following one:

- Block Substitution. This kind of update operation is applied on blocks $M_{i}$ and $M_{i}^{\prime}$, where $M_{i}^{\prime}$ is the changed version of the block $M_{i}$. In total two block hash operations are applied. The hash update operation is given by Algorithm 2, and its graphical presentation in Figure 5.

Data overhead. There is no data overhead in this case. The final hash value has a size of $k$ bits. This is the only data necessary to store if we want to recompute the hash.

2. Variable-size data. Hashing structured data which can have a variable size but where the data blocks always have a unique block identifier that does not change.

Block indexing. Data is divided into an ordered sequence of blocks $M_{1}, M_{2}, \ldots, M_{n}$. In this case the unique identifier consists of a nonce for that block, denoted as $B N$ and a pointer to the nonce of the next block i.e. $\left.I D_{i} \equiv\left(B N_{i}, p t r_{B N_{i+1}}\right)\right)$. Additionally, we need 


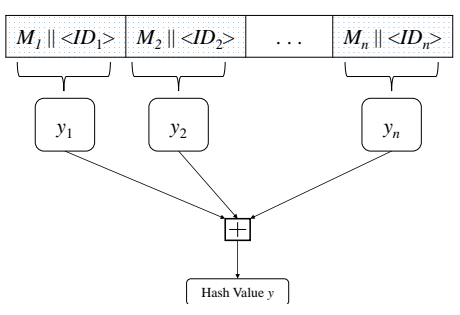

Fig. 2. Construction of incremental hash function for fixed size data.

\begin{tabular}{|c|}
\hline Alg \\
\hline $\begin{array}{l}\text { one } M_{i}^{\prime} \text {. } \\
\text { The old hash value } y \text {. }\end{array}$ \\
\hline $\begin{array}{l}\text { Output. } k \text { bits of updated hash out- } \\
\text { put. }\end{array}$ \\
\hline 1. Calculate $y_{i}=h\left(\overline{M_{i}}\right)$ \\
\hline 2. Calculate $y_{i}^{\prime}=h\left(\overline{M_{i}^{\prime}}\right)$ \\
\hline $\begin{array}{l}\text { 3. Combine } y, y_{i} \text { and } y_{i}^{\prime} \text { via a combin- } \\
\text { ing group operation } \bigoplus_{64} \text { to get the } \\
\text { new updated final hash value } y^{\prime}= \\
y \square_{64} y_{i} \bigoplus_{64} y_{i}^{\prime} \text {; }\end{array}$ \\
\hline 4 (1) \\
\hline
\end{tabular}

Fig. 4. An algorithm for incremental hash update operation: Block Substitution.

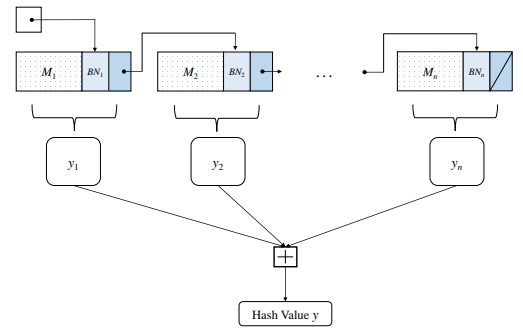

Fig. 3. Construction of incremental hash function for variable size data. The colored parts present the data overhead.

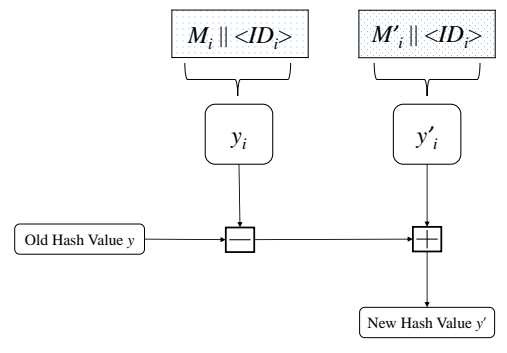

Fig. 5. An update hash operation: Block substitution. Note that when we deal with the fixed data size $I D_{i} \equiv\langle i\rangle$ and for variable data size it is $I D_{i} \equiv$ $\left(B N_{i}, p t r_{B N_{i+1}}\right)$.

a head for this data structure i.e., a pointer for the first data block $M_{1}$ and the pointer of the last block $M_{n}$, that points to NULL i.e. $p t r_{B N_{n+1}}=$ NULL. This hybrid data structure is, in fact, a singlylinked list with direct access via unique nonces and it is shown in Figure 3.

Incremental update operations. In this case, we have the following three update operations:

- Block Substitution. This kind of update operation is applied on block $M_{i}$ and $M_{i}^{\prime}$ (the changed version of the block $M_{i}$ ). The hash update operation is the same as in the case of fixed size data settings, just with a difference in the presentation of $I D$, i.e. $I D_{i}=\left(B N_{i}, p t r_{B N_{i+1}}\right)$. In total two block hash operations are applied. An algorithm is given by Algorithm 2 and its graphical presentation is given in Figure 5 . 
- Block Insertion. An insertion of a new block $M_{j}$ with nonce $B N_{j}$ after block $M_{i}$ is performed by changing the unique identifier $I D_{i}$. The old value of $I D_{i}=\left(B N_{i}, p t r_{B N_{i+1}}\right)$ is replaced by the new value $I D_{i}=\left(B N_{i}, p t r_{B N_{j}}\right)$. In total three block hash operations are applied. This operation is given by Algorithm 3, and its graphical presentation in Figure 8.

- Block Deletion. To delete a block $M_{i}$ we need to change the unique identifier of the $i$-1-th block, $I D_{i-1}=\left(B N_{i-1}, p t r_{B N_{i}}\right)$ into $I D_{i-1}=\left(B N_{i-1}, p t r_{B N_{i+1}}\right)$. In total three block hash operations are applied. The hash update operation is given by Algorithm 4, and its graphical presentation in Figure 9.

Data overhead. In this case, we have two sub-cases: (1): The size of the data that is hashed is tightly coupled with the media where it is stored. There is no data overhead, and the output is just $k$ bits of the final hash value. (2): The size of the data that is hashed is flexible. The data overhead is the information about the hybrid singly-linked list with direct access $I D_{1}, I D_{2}, \ldots, I D_{n}$ that is given together with the final hash value of size $k$ bits.

\begin{tabular}{|c|}
\hline Algorithm 3 - Block Insertion \\
\hline $\begin{array}{l}\text { Input. The block } M_{i} \text { and } I D_{i} \text { after which } \\
\text { the insertion will be done; The new block } \\
M_{j} ;\end{array}$ \\
\hline Output. $k$ bits of hash output. \\
\hline 1. Calculate $y_{i}=h\left(\overline{M_{i}}\right)$ \\
\hline 2. Calculate $y_{j}=h\left(\overline{M_{j}}\right)$ \\
\hline $\begin{array}{l}\text { 3. Transform } I D_{i} \text { into } I D_{i}^{\prime} \text { i.e. } I D_{i}^{\prime} \equiv \\
\left(B N_{i}, p t r_{B N_{j}}\right)\end{array}$ \\
\hline $\begin{array}{l}\text { 4. Calculate } y_{i}^{\prime}=h\left(\overline{M_{i}^{\prime}}\right) \text {, where } \overline{M_{i}^{\prime}}= \\
M_{i} \| I D_{i}^{\prime} ;\end{array}$ \\
\hline $\begin{array}{l}\text { 5. Combine } y, y_{i}, y_{j} \text { and } y_{i}^{\prime} \text { via a com- } \\
\text { bining group operation } \bigoplus_{64} \text { to get the } \\
\text { new updated final hash value } y^{\prime}= \\
y \square_{64} y_{i} \boxplus_{64} y_{i}^{\prime} \boxplus_{64} y_{j} ;\end{array}$ \\
\hline 4. Output $y$ and store it. \\
\hline
\end{tabular}

Fig. 6. An algorithm for incremental hash update operation in the variable size setting: Block Insertion. Here the block $M_{j}$ is inserted after the block $M_{i}$.

\begin{tabular}{|c|}
\hline Algorithm 4 - Block Deletion \\
\hline $\begin{array}{l}\text { deleted; The previous block } M_{i-1} \text { and } I D_{i-1} \\
\text { from the sequence; }\end{array}$ \\
\hline Outp \\
\hline 1. Calculate $y_{i-1}=h\left(\overline{M_{i-1}}\right)$ \\
\hline 2. Calculate $y_{i}=h\left(\overline{M_{i}}\right)$ \\
\hline $\begin{array}{l}\text { 3. Transform } I D_{i-1} \text { into } I D_{i-1}^{\prime} \text { as } I D_{i-1}^{\prime} \equiv \\
\left(B N_{i-1}, p t r_{B N_{i+1}}\right) ;\end{array}$ \\
\hline $\begin{array}{l}\text { 4. Calculate } y_{i-1}^{\prime}=h\left(\overline{M_{i-1}^{\prime}}\right) \text {, where } \overline{M_{i-1}^{\prime}}= \\
M_{i-1} \|\left(B N_{i-1}, p \operatorname{tr}_{B N_{i+1}}\right) ;\end{array}$ \\
\hline $\begin{array}{l}\text { 5. Combine } y, y_{i-1}, y_{i} \text { and } y_{i-1}^{\prime} \text { via a } \\
\text { combining group operation } \bigoplus_{64} \text { to get } \\
\text { the new updated final hash value } y^{\prime}= \\
y \square_{64} y_{i-1} \square_{64} y_{i} \bigoplus_{64} y_{i-1}^{\prime} ;\end{array}$ \\
\hline 4. Output \\
\hline
\end{tabular}

Fig. 7. An algorithm for incremental hash update operation in the variable size setting: Block Deletion. Here the block $M_{i}$ is deleted.

\subsection{Incremental tree based hash scheme}

Merkle proposed the tree hashing which can be used for incremental hashing [15]. In his scheme, the incrementality is implemented at the cost of 


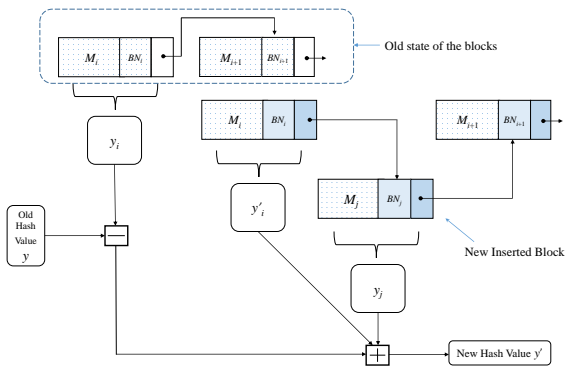

Fig. 8. An update hash operation: Block insertion. Here the block $M_{j}$ is inserted after the block $M_{i}$.

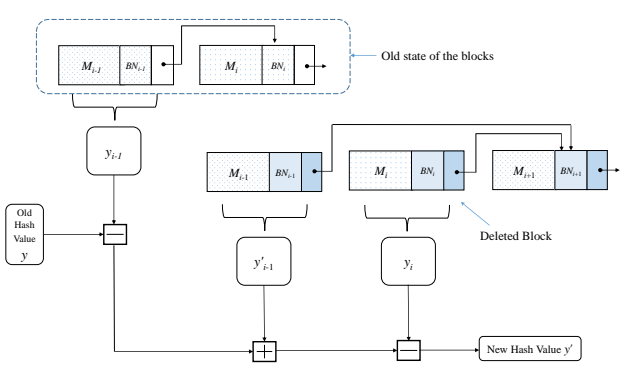

Fig. 9. An update hash operation: Block deletion. Here the block $M_{i}$ is deleted.

storing all intermediate hash values of all tree levels. This can significantly increase the data overhead. To reduce the data overhead we can limit the tree depth to one or two leves $[6,7]$. Assume for simplicity that the hash tree has depth 1 . The graphical representation of the one level tree hashing mode is given in Figure 12. An algorithmic description of the one level tree hashing is given by Algorithm 5 .

\begin{tabular}{|c|}
\hline $\begin{array}{c}\text { Algorithm } 5 \text { - One level tree } \\
\text { hashing }\end{array}$ \\
\hline $\begin{array}{l}\text { Input. A sequence of blocks } \\
M_{1}, M_{2}, \ldots, M_{n} \text { with fixed size of } \\
b \text { bits. }\end{array}$ \\
\hline $\begin{array}{l}\text { Output. } n * k \text { bits of leaves hashes and } \\
k \text { bits of the root hash. }\end{array}$ \\
\hline $\begin{array}{l}\text { 1. For each block } M_{i}, i=1, \ldots, n, \text { ap- } \\
\text { ply } h \text { to them to get a hash value } \\
y_{i}=h\left(M_{i}\right) ; \\
\text { 2. Concatenate } y_{1}, \ldots, y_{n} \text { and apply } h \\
\text { to the concatenated string to get the } \\
\text { root hash value }\end{array}$ \\
\hline $\begin{array}{l}y=h\left(y_{1} \| y_{2}, \ldots, y_{n}\right) . \\
\text { 3. Output } y \text { and store it. Store } \\
\text { all the intermediate leaves hashes } \\
y_{1}, y_{2}, \ldots, y_{n}\end{array}$ \\
\hline
\end{tabular}

Fig. 10. An algorithm for incremental tree based hash function with depth 1 .

\begin{tabular}{|c|}
\hline $\begin{array}{c}\text { Algorithm } 6 \text { - Block substitution in } \\
\text { tree hashing }\end{array}$ \\
\hline
\end{tabular}

Input. The position $i$ of the old block and the new one $M_{i}^{\prime}$.

The old hash value $y$ and all intermediate leaves hashes $y_{1}, y_{2}, \ldots, y_{n}$.

Output. $n * k$ bits of leaves hashes and $k$ bits of the root hash.

1. Calculate $y_{i}^{\prime}=h\left(M_{i}^{\prime}\right)$;

2. Replace $y_{i}$ with $y_{i}^{\prime}$;

3. Concatenate $y_{1}, \ldots, y_{n}$ and apply $h$ to the concatenated string to get the root hash value $y=h\left(y_{1} \| y_{2}, \ldots, y_{n}\right)$.

4. Output $y$ and store it. Store all the intermediate leaves hashes $y_{1}, y_{2}, \ldots, y_{n}$.

For this scheme, the data $M$ is divided into blocks $M_{1}, M_{2}, \ldots, M_{n}$ and we need the following components:

One level tree-based hash function. Any cryptographic hash function $h$ that maps data with arbitrary size into $k$ bits can be used. It has two stages: 
- Hashing tree leaves. The hash function $h$ maps the leaves $M_{i}$ of $b$ bits into $k$ bits i.e. $y_{i}=h\left(M_{i}\right)$.

- Root hash. The final hash value $y$ is computed by hashing the concatenation of the hashes of the leaves, i.e. $y=h\left(y_{1}\left\|y_{2}\right\| \ldots \| y_{n}\right)$.

Incremental update operation. Once the root hash is computed, the update operation has the following variants:

- Block Substitution. This kind of update operation is applied on blocks $M_{i}$ and $M_{i}^{\prime}$, where $M_{i}^{\prime}$ is a changed version of the block $M_{i}$. In total one block hash operation and one root hash computation are performed. This operation is given by Algorithm 6, and its graphical presentation in Figure 13.

- Block Insertion. An insertion of a new block $M_{j}$ after block $M_{i}$ means insertion of the new hash value $h\left(M_{j}\right)$ after the stored hash value $h\left(M_{i}\right)$ and computation of the root hash. This operation is given by Algorithm 7, and its graphical presentation in Figure 16.

- Block Deletion. To delete a block $M_{i}$ we need to delete the stored hash value of that block and to compute the root hash. It is given by Algorithm 8, and its graphical presentation in Figure 17.

Data overhead. The data overhead is $(n+1) \times k$ bits which come from $n$ hashes $y_{i}$ and the final root hash $y$.

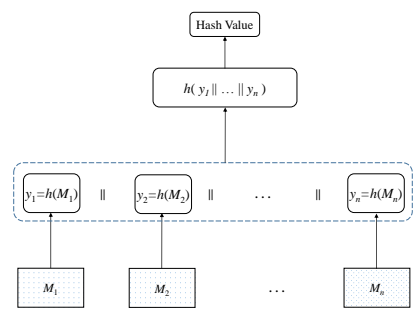

Fig. 12. Incremental hashing using one level tree structure.

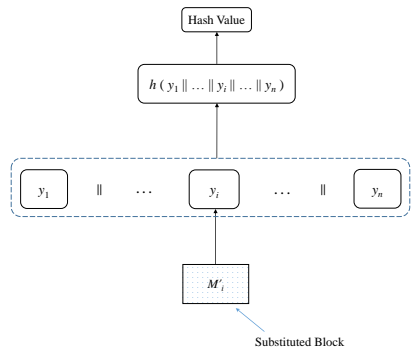

Fig. 13. An update hash operation: Block substitution. Here the block $M_{i}$ is substituted with the block $M_{i}^{\prime}$.

\section{Definition of $i$ SHAKE and Security analysis}

Recently, NIST proposed the DRAFT SHA-3 Standard: PermutationBased Hash and Extendable-Output Functions [17], containing definitions for two Extendable-Output Functions named SHAKE128 and SHAKE256. We just briefly mention their definitions:

$$
\begin{gathered}
\operatorname{SHAKE} 128(M, d)=\operatorname{RawSHAKE} 128(M \| 11, d), \text { where } \\
\operatorname{RawSHAKE} 128(M, d)=\operatorname{KECCAK}[256](M \| 11, d),
\end{gathered}
$$

and 


\begin{tabular}{|c|}
\hline $\begin{array}{c}\text { Algorithm } 7 \text { - Block insertion in } \\
\text { tree hashing }\end{array}$ \\
\hline $\begin{array}{l}\text { Input. The position } i \text { where the insert } \\
\text { should be done. } \\
\text { The new block } M_{j} \text { and all intermediate } \\
\text { leaves hashes } y_{1}, y_{2}, \ldots, y_{n} \text {. }\end{array}$ \\
\hline $\begin{array}{l}\text { Output. } n * k \text { bits of leaves hashes and } k \\
\text { bits of the root hash. }\end{array}$ \\
\hline $\begin{array}{l}\text { 1. Calculate } y_{j}=h\left(M_{j}\right) \\
\text { 3. Concatenate } y_{1}, \ldots, y_{i}, y_{j}, y_{i+1}, \ldots y_{n} \\
\text { and apply } h \text { to the concatenated string } \\
\text { to get the root hash value } y= \\
h\left(y_{1} \| y_{2}, \ldots, y_{n+1}\right) \text {. } \\
\text { 4. Output } y \text { and store it. Store all the in- } \\
\text { termediate leaves hashes } y_{1}, y_{2}, \ldots, y_{n+1} \text {. }\end{array}$ \\
\hline
\end{tabular}

Fig. 14. An algorithm for incremental tree based hash update operation: Block Insertion, where the block $M_{j}$ is inserted after the block $M_{i}$.

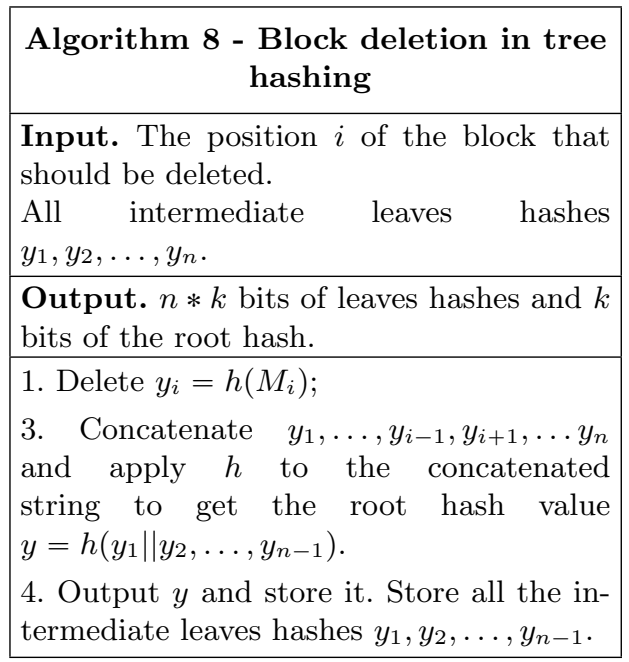

Fig. 15. An algorithm for incremental tree based hash update operation: Block Deletion. Here the block with index $i$ is deleted.

$$
\begin{gathered}
\operatorname{SHAKE} 256(M, d)=\operatorname{RawSHAKE} 256(M \| 11, d), \text { where } \\
\operatorname{RawSHAKE} 256(M, d)=\operatorname{KECCAK}[512](M \| 11, d) .
\end{gathered}
$$

$i$ SHAKE128 is the instantiation of the incremental hash function from Algorithm 1 (Section 3), where for the hash function $h$ we use SHAKE128 with the output size of 2688 up to 4160 bits. Similarly for $i$ SHAKE256 the output size is in the range of 6528 and 16512 bits.

Using appropriate values for the time complexity of Wagner's generalized birthday attack (Proposition 1), we have the following:

Proposition 2. Let for iSHAKE128 parameter $k=2688$ (for iSHAKE256, $k=6528$ ) and let the maximal allowed number of blocks be $N=2^{25}$ $\left(N=2^{28}\right.$ for iSHAKE256). Then

$$
\min _{n \leqslant N} O\left(n \cdot 2^{\frac{k}{1+\lg [n]}}\right)=2^{128.385} \quad\left(2^{253.103}\right) .
$$

By a simple multiplication $b \times N$ we have the following:

Proposition 3. The lower bound of $2^{128}$ on the complexity of Wagner's generalized birthday attack on iSHAKE128 for block sizes of $1 \mathrm{~KB}, 2 \mathrm{~KB}$ and $4 K B$ for the data blocks $M_{i}$, can be achieved by hashing files long 32 GB, $64 G B$ and $128 G B$ correspondingly. Also for the $2^{256}$ security bound for $i$ SHAKE256 for block sizes of $1 K B, 2 K B$ and $4 K B$ for the data blocks $M_{i}$, the hashing files should be long 256 GB, 512 GB and 1 TB correspondingly. 
It is normal to expect that $i$ SHAKE128 would be used for hashing files of size less than 32 GB. In this case there is a tradeoff between the security of finding second-preimage and the size of the hashed files which is expresses by the equation (3). For example, for small size files such as $160 \mathrm{~KB}$ the complexity of finding second-preimage is $2^{254}$ and for files of $1.25 \mathrm{~TB}$, the complexity drops down to $2^{112}$. Figure 18 shows that trade-off for different file sizes.

A similar reasoning applies to $i$ SHAKE256 for hashing files of size less than 256 GB. For example for file sizes of 1 MB the complexity of finding second-preimage is $2^{479}$ and for files of as much as 8 TB the complexity of finding collisions drop down to $2^{212}$. Figure 19 shows that trade-off for different file sizes.

If the length of the messages is not restricted, then the low bound security of $2^{128}$ or $2^{256}$ in equation (3) is achieved for messages with parameter values $k=4160$ bits for $i$ SHAKE128 and $k=16512$ bits for $i$ SHAKE256.

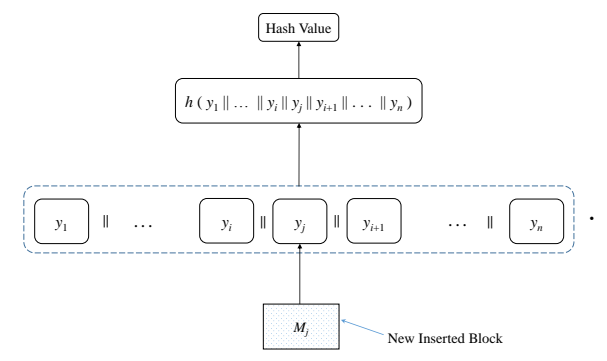

Fig. 16. An update hash operation: Block insertion. Here the block $M_{j}$ is inserted.

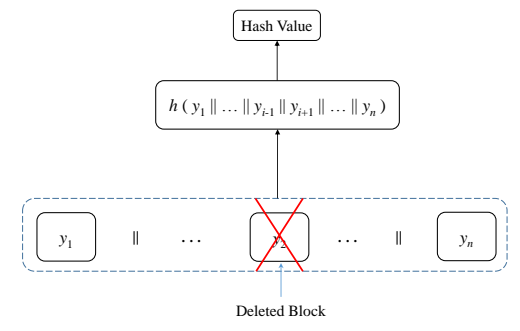

Fig. 17. An update hash operation: Block deletion. Here the block $M_{i}$ is deleted.

\section{Comparison Analysis}

To show the advantages of our new incremental schemes, we compared different performance aspects of our schemes with suitably chosen tree based hashing schemes. We note that a comparison of our approach to a sequential hashing mode does not make sense because it is not parallel and it is not incremental. The only fair comparison would be to schemes with these properties, and currently, tree hashing is the best known method for achieving incrementality. We compared the update effort for different operations and data overhead that introduces additional storage cost. The results in terms of the needed number of operations are given in Table 1.

We also compared the performance in terms of speed of $i$ SHAKE and one level tree hashing. Table 2 and Table 3 show an evident speed advantage of $i \mathrm{SHAKE}$ over the corresponding incremental tree hashing of as much as 5 to 6 orders of magnitude. The results in the two tables can 


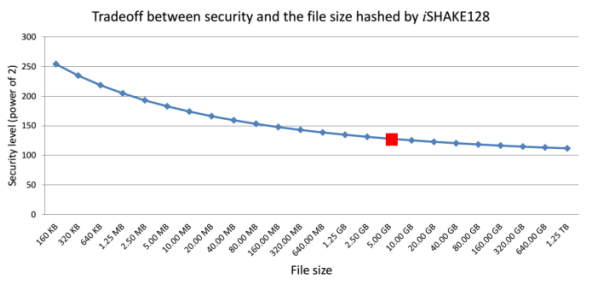

Fig. 18. A trade-off between finding collisions with the Wagner's generalized birthday attack and the size of the hashed file with $i$ SHAKE128

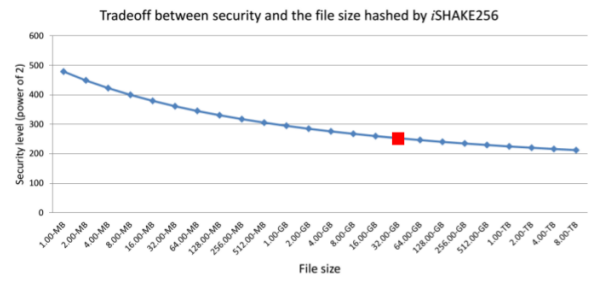

Fig. 19. A trade-off between finding collisions with the Wagner's generalized birthday attack and the size of the hashed file with $i$ SHAKE256

be interpreted as follows: For a fixed data overhead for both approaches, what amount of data should be digested in one incremental operation? If we assume an equal digest time per data byte, this can be directly translated into a speed comparison between the two. As an example, consider an input file of size 1MB. If we use $i$ SHAKE128 with blocks of $1 \mathrm{~KB}$, then the amount of bits that we need to store is just the output of the hash function or 2688 bits. If we bound the overhead to the same (or approximate) amount of bits for tree hashing, then we can split the message to a maximum of 10 blocks. In this case, each block will be of size 102.4KB. Thus, in case we have a change of few (up to several hundreds of bytes) that fall in one block of $1 \mathrm{~KB}, i \mathrm{SHAKE}$ will rehash only that small block of $1 \mathrm{~KB}$ while the tree version of SHA-3 will have to digest significantly bigger block of $102.4 \mathrm{~KB}$. This translates to speed advantage of $i$ SHAKE of 102.4 times.

\section{Conclusion}

The need for incremental hashing in the upcoming Zettabyte era is imminent. In this paper, we defined two incremental hash functions $i$ SHAKE128 and $i$ SHAKE256 with security level against collision attacks of 128 and 256 bits respectively. Both are based on the recent NIST proposal for SHA-3 Extendable-Output Functions SHAKE128 and SHAKE256. We presented constructions for two practical settings: fixed size data and variable size data. In the first one, our proposed scheme has an obvious advantage in the small overhead that it carries out, compared with any other tree-based hash scheme. Moreover, the speed-up is present even in the case where the same data overhead is used. In the second practical setting, our proposed scheme behaves approximately the same as tree based hashing when the dynamic data structure representing the unique identifier of the blocks should be stored. In the case where the unique identifiers of the data blocks are tightly coupled with the media where 


\begin{tabular}{|c|c|c|c|c|}
\hline $\begin{array}{c}\text { Incremental } \\
\text { Hashing Scenario }\end{array}$ & $\begin{array}{c}\text { Incremental } \\
\text { Operation }\end{array}$ & Update cost & Data overhead & $\begin{array}{c}\text { Collision between } \\
\text { parallel and } \\
\text { sequential } \\
\text { hashes } \\
\end{array}$ \\
\hline $\begin{array}{l}\text { Incremental hashing } \\
\text { in fixed size setting }\end{array}$ & $\begin{array}{l}\text { Block } \\
\text { Substitution }\end{array}$ & $\begin{array}{l}2 \text { data block hash } \\
\text { operations }\end{array}$ & $\begin{array}{l}k \text {-bits of hash output } \\
(2600 \leqslant k \leqslant 16000)\end{array}$ & No \\
\hline \multirow{3}{*}{$\begin{array}{l}\text { Incremental hashing } \\
\text { in variable size setting } \\
\text { (without migration) }\end{array}$} & \begin{tabular}{|l|} 
Block \\
Substitution
\end{tabular} & $\begin{array}{l}2 \text { data block hash } \\
\text { operations }\end{array}$ & \multirow{3}{*}{$\begin{array}{l}k \text {-bits of hash output } \\
(2600 \leqslant k \leqslant 16000)\end{array}$} & \multirow{3}{*}{ No } \\
\hline & \begin{tabular}{|l|} 
Block \\
Insertion
\end{tabular} & $\begin{array}{l}3 \text { data block hash } \\
\text { operations }\end{array}$ & & \\
\hline & \begin{tabular}{|l} 
Block \\
Deletion
\end{tabular} & $\begin{array}{c}3 \text { data block hash } \\
\text { operations }\end{array}$ & & \\
\hline Incremental hashing & \begin{tabular}{|l|} 
Block \\
Substitution
\end{tabular} & $\begin{array}{l}2 \text { data block hash } \\
\text { operations }\end{array}$ & \multirow{3}{*}{$\begin{array}{c}k \text {-bits of hash output } \\
(2600 \leqslant k \leqslant 16000) \\
+ \\
n \times 64 \text { bits for the } \\
\text { data structure }\end{array}$} & \multirow{3}{*}{ No } \\
\hline $\begin{array}{l}\text { in variable size setting } \\
\text { (with migration) }\end{array}$ & $\begin{array}{l}\text { Block } \\
\text { Insertion }\end{array}$ & $\begin{array}{l}3 \text { data block hash } \\
\text { operations }\end{array}$ & & \\
\hline & \begin{tabular}{|l|} 
Block \\
Deletion
\end{tabular} & $\begin{array}{l}3 \text { data block hash } \\
\text { operations }\end{array}$ & & \\
\hline \multirow{3}{*}{$\begin{array}{l}\text { Incremental tree } \\
\text { hashing with a tree } \\
\text { depth of } 1\end{array}$} & $\begin{array}{l}\text { Block } \\
\text { Substitution }\end{array}$ & $\begin{array}{l}1 \text { data block hash operation } \\
+1 \text { hash operation on the } \\
\text { intermediate leaves hashes }\end{array}$ & \multirow{3}{*}{$\begin{array}{c}n \times k \text { bits of intermediate } \\
\text { hash values } \\
+ \\
k \text { bits of final hash output } \\
= \\
(n+1) \times k \text { bits } \\
(160 \leqslant k \leqslant 512)\end{array}$} & \multirow[t]{3}{*}{ Yes [14] } \\
\hline & $\begin{array}{l}\text { Block } \\
\text { Insertion }\end{array}$ & \begin{tabular}{|c|}
1 data block hash operation \\
+1 hash operation on the \\
intermediate leaves hashes
\end{tabular} & & \\
\hline & \begin{tabular}{|l|} 
Block \\
Deletion
\end{tabular} & $\begin{array}{c}1 \text { hash operation on the } \\
\text { intermediate leaves hashes }\end{array}$ & & \\
\hline
\end{tabular}

Table 1. Comparison analysis between our incremental hash function approach and tree based hashing.

\begin{tabular}{|c|c|c|c|c|c|c|c|c|c|c|c|c|}
\hline \multicolumn{1}{|c|}{ Fixed data overhead of 2688 bits (iSHAKE128) and 2816 bits (SHA3-256 One Level Tree) } \\
\hline & \multicolumn{3}{|c|}{$1 \mathrm{MB}$} & \multicolumn{3}{|c|}{ 10MB } & \multicolumn{3}{c|}{$100 \mathrm{MB}$} & \multicolumn{5}{c|}{$1 \mathrm{~GB}$} \\
\hline Block size in KB & 1 & 4 & 8 & 1 & 4 & 8 & 1 & 4 & 8 & 1 & 4 & 8 \\
\hline $\begin{array}{c}\text { Speed advantage } \\
\text { (times) }\end{array}$ & 102.4 & 25.6 & 12.8 & 1024 & 256 & 128 & 10240 & 2560 & 1280 & 104857.6 & 26214.4 & 13107.2 \\
\hline
\end{tabular}

Table 2. Speed advantage of $i$ SHAKE128 in comparison with SHA3-256 one level tree-based hashing scheme when one block is updated

\begin{tabular}{|c|c|c|c|c|c|c|c|c|c|c|c|c|}
\hline \multicolumn{13}{|c|}{ Fixed data overhead of 6528 bits ( $i$ SHAKE256) and 6656 bits (SHA3-512 One Level Tree) } \\
\hline & \multicolumn{3}{|c|}{$1 \mathrm{MB}$} & \multicolumn{3}{|c|}{$10 \mathrm{MB}$} & \multicolumn{3}{|c|}{$100 \mathrm{MB}$} & \multicolumn{3}{|c|}{$1 \mathrm{~GB}$} \\
\hline Block size in $\mathrm{KB}$ & 1 & 4 & 8 & 1 & 4 & 8 & 1 & 4 & 8 & 1 & 4 & 8 \\
\hline \begin{tabular}{|c|} 
Speed advantage \\
(times)
\end{tabular} & 85.3 & 21.3 & 10.7 & 853.3 & 213.3 & 106.7 & 8533.3 & 2133.3 & 1066.7 & 87381.3 & 21845.3 & 10922.7 \\
\hline
\end{tabular}

Table 3. Speed advantage of $i$ SHAKE256 in comparison with SHA3-512 one level tree-based hashing scheme when one block is updated

they are stored, the situation is the same as in the fixed size setting. That is, again, our schemes show much better performance than tree hashing.

We believe that our work will be more than interesting for those practitioners who struggle from using incremental hashing because of the big data overhead that they need to take care of. Therefore, we leave the practical implementation of our newly defined schemes as a future work one that would possibly focus on some file system and using its structure practically without additional overhead to implement the incrementality of the scheme.

\section{References}

1. Amazon web services. An Amazon Company, 2015. http://aws . amazon.com/ec2/ instance-types/. 
2. Virtual machine and cloud service sizes for azure. Microsoft, 2015. https://msdn. microsoft.com/en-us/library/azure/dn197896.aspx.

3. Historical cost of computer memory and storage. hblok.net $\bullet$ Freedom, Electronics and Tech, February 2013. http://hblok.net/blog/storage/.

4. Mihir Bellare, Oded Goldreich, and Shafi Goldwasser. Incremental cryptography: The case of hashing and signing. In Yvo Desmedt, editor, CRYPTO, LNCS, pages 216-233. Springer, 1994.

5. Mihir Bellare and Daniele Micciancio. A new paradigm for collision-free hashing: Incrementality at reduced cost. In Walter Fumy, editor, EUROCRYPT, LNCS, pages 163-192. Springer, 1997.

6. Guido Bertoni, Joan Daemen, Michal Peeters, and Gilles Van Assche. Sakura: A flexible coding for tree hashing. In Ioana Boureanu, Philippe Owesarski, and Serge Vaudenay, editors, Applied Cryptography and Network Security, LNCS, pages 217-234. Springer International Publishing, 2014.

7. Guido Bertoni, Joan Daemen, Michal Peeters, and Gilles Van Assche. Sufficient conditions for sound tree and sequential hashing modes. International Journal of Information Security, (4):335-353, 2014.

8. Fay Chang, Jeffrey Dean, Sanjay Ghemawat, Wilson C. Hsieh, Deborah A. Wallach, Mike Burrows, Tushar Chandra, Andrew Fikes, and Robert E. Gruber. Bigtable: A distributed storage system for structured data. In Proceedings of the 7th USENIX Symposium on Operating Systems Design and Implementation - vol. 7, OSDI '06, pages 15-15, Berkeley, CA, USA, 2006. USENIX Association.

9. Cisco. Cisco visual networking index: Forecast and methodology, 2012-2017. White Paper, May 2013.

10. EMC. The EMC Digital Universe study with research and analysis by IDC. Open Report, April 2014.

11. Danilo Gligoroski and Simona Samardjiska. iSHAKE: Incremental Hashing with SHAKE128 and SHAKE256 for the Zettabyte Era. SHA3 Workshop, 2014. http://csrc.nist.gov/groups/ST/hash/sha-3/Aug2014/ documents/gligoroski\_paper\_sha3\_2014\_workshop.pdf.

12. Jane K. Hart and Kirk Martinez. Environmental sensor networks: A revolution in the earth system science? Earth-Science Reviews, 78(34):177 - 191, 2006.

13. Mark Hornby. Review Of The Best Cloud Storage Services. http://www . thetop10bestonlinebackup.com/cloud-storage, 2015. Online, accessed 01 March 2016.

14. John Kelsey. What Should Be In A Parallel Hashing Standard? NIST, 2014 SHA3 Workshop. Available at http://csrc.nist.gov/groups/ST/hash/sha-3/ Aug2014/documents/kelsey_sha3_2014_panel.pdf.

15. Ralph C. Merkle. A digital signature based on a conventional encryption function. In Advances in Cryptology, CRYPTO '87, pages 369-378, London, UK, 1988. Springer-Verlag.

16. Mike S. How are digital movies distributed and screened? Every question answered! http://goo.gl/qLYoIV. Online, accessed 01 March 2016.

17. NIST. DRAFT SHA-3 Standard: Permutation-Based Hash and ExtendableOutput Functions. FIPS 202, April 2014.

18. National Centers for Environmental Information NOAA. Climate Forecast System Version 2 (CFSv2). Available at https://www.ncdc.noaa.gov/data-access/ model-data/model-datasets/climate-forecast-system-version2-cfsv2.

19. David Wagner. A generalized birthday problem. In Moti Yung, editor, CRYPTO, LNCS, pages 288-303. Springer, 2002. 